\title{
Removing Lipemia in Serum/Plasma Samples: A Multicenter Study
}

María-José Castro-Castro Đi, Ph.D. ${ }^{1}$, Beatriz Candás-Estébanez, Ph.D. ${ }^{1}$, Margarita Esteban-Salán, Ph.D. ${ }^{2}$, Pilar Calmarza, Ph.D. ${ }^{3}$, Teresa Arrobas-Velilla, Ph.D. ${ }^{4}$, Carlos Romero-Román, Ph.D. ${ }^{5}$, Miguel Pocoví-Mieras, Ph.D. ${ }^{6}$, José-Ángel Aguilar-Doreste, Ph.D. , on behalf of the Commission on Lipoprotein and Vascular Diseases, Sociedad Española de Química Clínica

${ }^{1}$ Clinical Laboratory, Bellvitge University Hospital, Barcelona, Spain; ${ }^{2}$ Biochemitry Service, Cruces University Hospital, Vizcaya, Spain; ${ }^{3}$ Laboratory of Clinical Biochemistry, Miguel Servet University Hospital, Zaragoza, Spain; ${ }^{4}$ Laboratory of Clinical Biochemistry, Virgen del Rocío University Hospital, Sevilla, Spain; ${ }^{5}$ Laboratory of Clinical Biochemistry, Albacete Hospital, Albacete, Spain; ${ }^{6}$ Department of Biochemistry and Molecular and Cellular Biology, University of

Zaragoza, Zaragoza, Spain; 'Laboratory of Clinical Biochemistry, Gran Canaria Dr. Negrín University Hospital, Las Palmas de Gran Canaria, Spain

Background: Lipemia, a significant source of analytical errors in clinical laboratory settings, should be removed prior to measuring biochemical parameters. We investigated whether lipemia in serum/plasma samples can be removed using a method that is easier and more practicable than ultracentrifugation, the current reference method.

Methods: Seven hospital laboratories in Spain participated in this study. We first compared the effectiveness of ultracentrifugation $(108,200 \times g)$ and high-speed centrifugation $(10,000 \times g$ for 15 minutes) in removing lipemia. Second, we compared high-speed centrifugation with two liquid-liquid extraction methods-LipoClear (StatSpin, Norwood, USA), and 1,1,2-trichlorotrifluoroethane (Merck, Darmstadt, Germany). We assessed 14 biochemical parameters: serum/plasma concentrations of sodium ion, potassium ion, chloride ion, glucose, total protein, albumin, creatinine, urea, alkaline phosphatase, gammaglutamyl transferase, alanine aminotransferase, aspartate-aminotransferase, calcium, and bilirubin. We analyzed whether the differences between lipemia removal methods exceeded the limit for clinically significant interference (LCSI).

Results: When ultracentrifugation and high-speed centrifugation were compared, no parameter had a difference that exceeded the LCSI. When high-speed centrifugation was compared with the two liquid-liquid extraction methods, we found differences exceeding the LCSI in protein, calcium, and aspartate aminotransferase in the comparison with 1,1,2-trichlorotrifluoroethane, and in protein, albumin, and calcium in the comparison with LipoClear. Differences in other parameters did not exceed the LCSI.

Conclusions: High-speed centrifugation (10,000 $\times$ g for 15 minutes) can be used instead of ultracentrifugation to remove lipemia in serum/plasma samples. LipoClear and 1,1,2-trichlorotrifluoroethane are unsuitable as they interfere with the measurement of certain parameters.

Key Words: Lipemia, Interference, Lipid removal method, High-speed centrifugation, LipoClear, 1,1,2-trichlorotrifluoroethane
Received: November 12, 2017

Revision received: January 30, 2018

Accepted: June 20, 2018

\section{Corresponding author:}

María-José Castro-Castro

(iD) https://orcid.org/0000-0002-6080-3716

Bellvitge University Hospital, C/Feixa Llarga s/n, L'Hospitalet de Llobregat, Barcelona,

C.P. 08907 , Spain

Tel: +349-3260-7573

E-mail: mjcastro@bellvitgehospital.cat

\section{INTRODUCTION}

Lipemia is defined as visible turbidity in serum or plasma sam- ples due to the presence of lipoprotein particles, especially chylomicrons. The most common cause of turbidity is a high concentration of triglycerides [1, 2]. Lipemia can be a significant 
source of analytical errors in clinical laboratory settings, which may result in serious diagnostic consequences. It is the most common interference in outpatient populations, occurring in up to $7 \%$ of samples [3].

The most common pre-analytical cause of lipemia is usually non-compliance to fasting prior to sampling [4]. Further, some pathological conditions may increase the turbidity of samples because of an increase in the concentration of triglycerides; these include alcohol abuse, diabetes mellitus, hypothyroidism, pancreatitis, and certain treatments, including protease inhibitors (in HIV infection), estrogens, and contraceptives [5, 6].

Lipemia interferes with measurements of biological parameters (sodium ion, transaminases, etc.) by changing the dispersion or absorption of light and even producing volume depletion in the aqueous phase, increasing the non-aqueous volume instead [7]. The turbidity produced by high lipids in samples mainly affects spectrophotometric methods due to the decrease in intensity of light reaching the solution [4, 8]. One example of the solvent displacement effect is pseudohyponatremia, a wellrecognized effect resulting from sodium remaining in the aqueous phase of the sample [9].

In clinical laboratory settings, it is important to remove lipemia by using an appropriate method. Sample ultracentrifugation is the recommended method according to the CLSI [10]. However, because of high cost, the necessary equipment is not available in many laboratories. Therefore, less potent centrifugation methods to remove lipoproteins and eliminate turbidity have been studied. Studies have reported no statistically significant differences in the serum concentrations of sodium ion, creatinine, urate, proteins, lactate dehydrogenase, and magnesium in lipemic serum after high-speed centrifugation at $10,000 \times g$ or ultracentrifugation at $100,000 \times g[8,9]$. Accordingly, it is important to determine if there are other easier methods to remove lipemia in common laboratory samples.

In addition to centrifugation methods, there are chemical methods for lipemia removal. For example, polar solvents can be used to separate lipids from the aqueous phase in lipemic samples based on phase differences. One of these methods uses the commercial product LipoClear (StatSpin, Norwood, Massachusetts, USA), which contains a non-ionic, non-toxic polymer that binds to the lipids, and after centrifugation, the concentration of biochemicals in the supernatant can be measured. However, LipoClear interferes with the measurement of several biochemical parameters, including gamma-glutamyl transferase, C-reactive protein, and creatine kinase 2 [11, 12]. Another product used for lipemia removal is 1,1,2-trichlorotriflu- oroethane. This chlorofluorocarbon is used as an organic solvent with polar properties, which allows the separation of lipids by liquid-liquid phase extraction.

We investigated whether lipemia in serum/plasma samples can be removed using a method that is easier and more practicable than ultracentrifugation, focusing on high-speed centrifugation and liquid-liquid extraction using either LipoClear or 1,1,2trichlorotrifluoroethane. Our study is novel in that it evaluated the interference of chemical processes during lipemia removal for measuring biochemical parameters in plasma/serum samples, using high-speed centrifugation as a reference method. We assessed the suitability of chemical methods that do not require the use of a high-speed centrifuge in the laboratory.

\section{METHODS}

\section{Study design and biochemical analyses}

This was a prospective and transversal study involving seven hospital laboratories in Spain: Gran Canaria Dr. Negrín University Hospital (Las Palmas de Gran Canaria), Bellvitge University Hospital (Barcelona), Corporació Sanitària Parc Taulí (BarceIona), Cruces University Hospital (Vizcaya), Virgen del Rocío University Hospital (Sevilla), Albacete Hospital (Albacete), and Miguel Servet University Hospital (Zaragoza). Gran Canaria Dr. Negrín University Hospital participated in phase 1 of the study. A minimum of 36 lipemic samples from the other six laboratories were planned to be included from January 2013 to January 2014.

Samples were randomly selected from those processed routinely in each laboratory over the study period. They were obtained from patients who visited the hospitals for scheduled analysis. No extra clinical procedures other than the scheduled ones were performed. Biochemical parameters were measured in ISO 9001-certified laboratories that followed Westgard quality control rules (calibration procedures were performed when required). Institutional Review Board approval was obtained at each hospital.

\section{1) Phase 1: Comparison of ultracentrifugation and high-speed centrifugation}

In this phase, lipemic samples were processed in the clinical laboratory of the Gran Canaria Dr. Negrín University Hospital by ultracentrifugation and by high-speed centrifugation, followed by biochemical analyses. Thirty-two lipemic serum samples randomly selected from patients who had undergone a blood test in the laboratory were included. The triglyceride concentration 
of these samples was between 900 and 4,300 mg/dL. The samples were processed on the day of collection or were stored at $2-8^{\circ} \mathrm{C}$ for less than one week in case they could not be processed immediately. Serum samples were homogenized with a vortex mixer (Thermolyne, Dubuque, lowa, USA); $1.5 \mathrm{~mL}$ and 1 $\mathrm{mL}$ were separated for the ultracentrifugation and high-speed centrifugation methods, respectively.

For ultracentrifugation, the $1.5-\mathrm{mL}$ sample was pipetted into a $13 \times 51-\mathrm{mm}$ polycarbonate tube and centrifuged in a Beckman TL-100 ultracentrifuge at $108,200 \times g$ for 20 minutes at $6^{\circ} \mathrm{C}$ (Beckman Instruments, Fullerton, California, USA), using a fixedangle TLA 100.3 rotor. After ultracentrifugation, the infranatant was collected using a fine-needle syringe.

For high-speed centrifugation, the 1-mL sample was centrifuged at $10,000 \times g$ for 10 minutes in a high-speed centrifuge (Abbott Heraeus Biofuge, Willowbrook, Illinois, USA). The aqueous phase was collected at the bottom of the tube, using a fineneedle syringe with care not to aspirate the non-aqueous phase.

Concentrations of the following biochemical parameters were measured using a Cobas c701 analyzer (Roche Diagnostics, Mannheim, Germany): triglycerides by the lipoprotein-lipase/oxidase/peroxidase method; sodium ion, potassium ion, and chloride ion by indirect potentiometry; glucose by the hexokinase method; total protein by the Biuret method; albumin by binding of cromocresol green; creatinine by the Jaffe method; urea by the urease method; alkaline phosphatase, gamma-glutamyl transferase, alanine aminotransferase, and aspartate-aminotransferase by methods recommended by the International Federation of Clinical Chemistry (IFCC) [13-16]; calcium by the ocresolphthalein complexone method; and bilirubin by the 3,5-dichlorophenyl diazonium method.

2) Phase 2: Comparison of three methods of lipemia removal.

The other six participating laboratories tested a minimum of 12 samples with triglyceride concentrations $<750 \mathrm{mg} / \mathrm{dL}$ (8.55 $\mathrm{mmol} / \mathrm{L}), 12$ samples with triglyceride concentrations of 750 $1,500 \mathrm{mg} / \mathrm{dL}$ (8.55-17.1 mmol/L), and 12 samples with triglyceride concentrations $>1,500 \mathrm{mg} / \mathrm{dL}(17.1 \mathrm{mmol} / \mathrm{L})$. The samples were processed on the day of collection or were stored at 2 $-8^{\circ} \mathrm{C}$ for less than one week in case they could not be processed immediately. Serum or plasma samples were homogenized.

The following lipid removal methods were used in this phase: (1) high-speed centrifugation: $1 \mathrm{~mL}$ of serum/plasma sample in an Eppendorf tube was centrifuged for 15 minutes at 10,000 ×g. The lower aqueous phase was collected using a fine-needle sy- ringe, being careful not to aspirate the lipid phase. (2) (StatSpin, Norwood, Massachusetts, USA): in an Eppendorf tube, $0.5 \mathrm{~mL}$ of sample and $0.1 \mathrm{~mL}$ of the LipoClear reagent were added, left to stand for 10 minutes, and mixed well. The mixture was centrifuged at 2,000 $\times g$ for 20 minutes, and the clarified supernatant was collected for analysis. All results were multiplied by 1.2 to compensate for the dilution of the original sample, according to the manufacturer's instruction. (3) 1,1,2-trichlorotrifluoroethane (Merck, Darmstadt, Germany): in a glass tube, $1 \mathrm{~mL}$ of sample and $0.7 \mathrm{~mL}$ of 1,1,2-trichlorotrifluoroethane were added, homogenized vigorously for 30 seconds on a shaker to ensure complete homogenization, and centrifuged for 10 minutes at $2,000 \times g$. The supernatant was collected for analysis.

In total, 14 biochemical parameters were studied (and concentration of triglycerides in serum/plasma was determined to verify that they were lipemic samples). All parameters were frequently requested in the clinical laboratories participating in the study. The presence of chylomicrons (sodium and potassium ions) is known to interfere with the measurement of certain selected parameters (sodium ion, transaminases, etc.). Concentrations of the biochemical parameters were measured using the same methods as in Phase 1, on one of the following systems: AU5400 (Olympus, Tokyo, Japan), Cobas c711 (Roche Diagnostics), Cobas c701 (Roche Diagnostics), or ADVIA 2400 (Siemens, Munich, Germany).

\section{Statistical analysis}

Grubbs test was used to detect aberrant results. The KolmogorovSmirnov test was used to analyze data distribution. Parametric and non-parametric data were summarized as means with standard deviations or medians with interquartile range, and compared using the paired t-test or Wilcoxon test, respectively (significance threshold: $P<0.05)$. Statistical analyses were performed using SPSS version 17.0 (Armonk, New York, USA).

For phase 1 , differences in the mean or median obtained from ultracentrifugation and high-speed centrifugation were analyzed to determine if the limit for clinically significant interference (LCSI) was exceeded.

The LCSI was calculated according to the formula recommended by the Sociedad Española de Bioquímica Clínica y Patología Molecular [17]:

\section{$\mathrm{LCSI}=(\mathrm{CV} b i$ / 2) $\bullet(\mathrm{Xc} / \mathrm{100})$,}

where $\mathrm{CVbi}$ is the coefficient of intra-individual biological variation obtained from CLSI [18] and $X_{C}$ is the critical or clinical decision concentration of the parameter.

For phase 2, we analyzed means or medians of biochemical 
parameters from high-speed centrifugation and LipoClear and

ane, to determine if differences exceeded the LCSI.

from high-speed centrifugation and 1,1,2-trichlorotrifluoroeth-

Table 1. Comparison between ultracentrifugation and high-speed centrifugation for removing lipemia in serum/plasma samples ( $\mathrm{N}=32$ )

\begin{tabular}{|c|c|c|c|c|}
\hline Biochemical parameter & $\begin{array}{c}\text { Mean (SD) } \\
\text { Ultracentrifugation }\end{array}$ & $\begin{array}{l}\text { Mean (SD) High-speed } \\
\text { centrifugation }\end{array}$ & $P^{*}$ & LCSI \\
\hline Sodium ion; c.sust. (mmol/L) & $141.52(4.59)$ & $141.03(4.88)$ & $<0.05$ & 1.27 \\
\hline Potassium ion; c.sust. (mmol/L) & $4.66(0.64)$ & $4.98(0.68)$ & 0.52 & 0.34 \\
\hline Chloride ion; C.sust. (mmol/L) & $102.63(4.96)$ & $102.31(5.17)$ & $<0.05$ & 0.61 \\
\hline Glucose; c.sust. (mmol/L) & $10.00(5.13)$ & $9.93(5.05)$ & $<0.05$ & 0.28 \\
\hline Protein; C.mass (g/L) & $73.58(4.33)$ & $73.22(4.49)$ & 0.09 & 0.99 \\
\hline Albumin; c.mass (g/L) & $44.00(1.60)$ & $43.75(1.67)$ & 0.35 & 0.68 \\
\hline Creatinine; c.sust. ( $\mu \mathrm{mol} / \mathrm{L})$ & $77.26(21.48)$ & $76.93(21.57)$ & 0.22 & 1.77 \\
\hline Urea; c.sust. (mmol/L) & $35.53(13.19)$ & $35.41(13.03)$ & 0.40 & 3.97 \\
\hline Alkaline phosphatase; c.cat. ( $\mu$ kat/L) & $1.46(0.36)$ & $1.46(0.36)$ & 0.16 & 0.048 \\
\hline Gamma-glutamyl transferase; c.cat. ( $\mu$ kat/L) & $1.44(1.49)$ & $1.43(1.50)$ & 0.08 & 0.988 \\
\hline Calcium; c.sust. (mmol/L) & $2.41(0.11)$ & $2.41(0.11)$ & 0.94 & 0.09 \\
\hline Bilirubin; c.sust. ( $\mu \mathrm{mol} / \mathrm{L})$ & $4.96(2.37)$ & $4.59(2.21)$ & $<0.05$ & 0.684 \\
\hline Alanine-aminotransferase; c.cat. ( $\mu$ kat/L) & $0.61(0.32)$ & $0.59(0.30)$ & $<0.05$ & 0.073 \\
\hline Aspartate-aminotransferase; c.cat. ( $\mu$ kat/L) & $0.47(0.24)$ & $0.47(0.24)$ & 0.38 & 0.028 \\
\hline
\end{tabular}

*This value was calculated using a paired t-test.

Abbreviations: LCSI, limit for clinically significant interference; c.sust., concentration of substance; c.cat., catalytic concentration.

Table 2. Comparison between liquid-liquid lipemia removal methods (LipoClear and 1,1,2-trichlorotrifluoroethane) and high-speed centrifugation for removing lipemia in serum/plasma samples

\begin{tabular}{|c|c|c|c|c|c|c|c|c|}
\hline \multirow[b]{2}{*}{ Biochemical parameter } & \multicolumn{4}{|c|}{ LipoClear } & \multicolumn{4}{|c|}{ 1,1,2-trichlorotrifluoroethane } \\
\hline & $\begin{array}{c}\text { Mean }(S D)^{*} \text { or } \\
\text { Median (IQR) }\end{array}$ & $\mathrm{N}$ & $P^{\dagger}$ & Exceeds LCSI & $\begin{array}{c}\text { Mean }(\mathrm{SD})^{*} \text { or } \\
\text { Median (IQR) }\end{array}$ & $\mathrm{N}$ & $P^{\dagger}$ & Exceeds LCSI \\
\hline Sodium ion; c.sust. (mmol/L) & $140.40(29.40)$ & 122 & $<0.05$ & NO & $133.09(15.31)^{*}$ & 130 & 0.07 & NO \\
\hline Potassium ion; c.sust. (mmol/L) & $4.55(0.74)^{*}$ & 120 & $<0.05$ & NO & $4.87(0.64)^{*}$ & 135 & 0.82 & NO \\
\hline Chloride ion; c.sust. (mmol/L) & $99.82(24.41)$ & 153 & $<0.05$ & NO & $103.95(7.88)$ & 156 & $<0.05$ & NO \\
\hline Glucose; c.sust. (mmol/L) & $6.80(5.72)$ & 156 & $<0.05$ & NO & $6.83(6.00)$ & 160 & $<0.05$ & NO \\
\hline Protein; c.mass (g/L) & $65.39(9.45)^{*}$ & 156 & $<0.05$ & YES & $73.31(6.30)^{*}$ & 160 & $<0.05$ & YES \\
\hline Albumin; c.mass (g/L) & $43.30(6.53)^{*}$ & 122 & $<0.05$ & YES & $43.35(4.91)^{*}$ & 126 & $<0.05$ & NO \\
\hline Creatinine; c.sust. ( $\mu \mathrm{mol} / \mathrm{L})$ & $79.29(36.24)$ & 157 & $<0.05$ & NO & $84.86(35.36)$ & 160 & 0.150 & NO \\
\hline Urea; c.sust. (mmol/L) & $37.78(19.08)$ & 157 & 0.07 & NO & $38.00(16.80)$ & 161 & $<0.05$ & NO \\
\hline Alkaline phosphatase; c.cat. ( $\mu$ kat/L) & $1.42(0.69)$ & 121 & 0.05 & NO & $1.42(0.72)$ & 122 & $<0.05$ & NO \\
\hline Gamma-glutamyl transferase; c.cat. ( $\mu$ kat/L) & $1.27(1.28)$ & 112 & $<0.05$ & NO & $1.32(1.5)$ & 110 & 0.57 & NO \\
\hline Calcium; c.sust. (mmol/L) & $2.22(0.66)$ & 143 & $<0.05$ & YES & $2.38(0.34)$ & 142 & $<0.05$ & YES \\
\hline Bilirubin; c.sust. ( $\mu \mathrm{mol} / \mathrm{L})$ & $4.96(3.42)$ & 143 & $<0.05$ & NO & $4.45(3.59)$ & 155 & $<0.05$ & NO \\
\hline Alanine-aminotransferase; c.cat. ( $\mu$ kat/L) & $0.43(0.33)$ & 157 & $<0.05$ & NO & $0.53(0.39)$ & 161 & $<0.05$ & NO \\
\hline Aspartate-aminotransferase; c.cat. ( $\mu$ kat/L) & $0.42(0.29)$ & 155 & $<0.05$ & NO & $0.48(0.37)$ & 160 & $<0.05$ & YES \\
\hline
\end{tabular}

*This value followed a parametric distribution.

${ }^{\dagger}$ This value was calculated using a paired t-test or Wilcoxon test.

Abbreviations: IQR, interquartile range; LCSI, limits for clinically significant interference; c.sust., concentration of substance; c.cat., catalytic concentration. 


\section{RESULTS}

Table 1 shows the results obtained in phase 1 . Data for all biochemical parameters were normally distributed. Statistically significant differences were found between ultracentrifugation and high-speed centrifugation for five parameters, but the differences in the means did not exceed the LCSI.

Table 2 shows the phase 2 results. Owing to missing results, 110-160 samples could be processed successfully. Differences in the following biochemical parameters did not exceed the LCSI in either comparison: sodium ion, potassium ion, chloride ion, glucose, creatinine, urea, alkaline phosphatase, gamma-glutamyl transferase, bilirubin, and alanine aminotransferase. Differences exceeded the LCSI for protein, calcium, and aspartate aminotransferase in the comparison of high-speed centrifugation and 1,1,2-trichlorotrifluoroethane. Differences exceeded the LCSI for protein, albumin, and calcium in the comparison of high-speed centrifugation and LipoClear.

\section{DISCUSSION}

We investigated whether a method that is easier and more practicable than ultracentrifugation can be used to remove lipemia in serum/plasma samples. A large number of biochemical parameters were tested, and the methods most commonly used for lipemia removal in laboratories were compared.

We found no statistically significant differences between ultracentrifugation and high-speed centrifugation for potassium ion, protein, albumin, creatinine, urea, alkaline phosphatase, calcium, gamma-glutamyl transferase, and aspartate aminotransferase. Sodium ion, chloride ion, glucose, bilirubin, and alanineaminotransferase showed statistically significant differences, but none of these exceeded the LCSI. Thus, the current reference method, ultracentrifugation, can be replaced with high-speed centrifugation at $10,000 \times g$ for 15 minutes. Analysis of the lowest fraction at the bottom would be suitable for measuring the concentrations of the biochemical parameters included in this study.

High-speed centrifugation is easy to perform in the laboratory, even in the emergency laboratory, where lipemic samples often arrive. It does not involve the introduction of any reagent that may interfere with the measurements, and only requires a highspeed centrifuge that reaches $10,000 \times$ g. In accordance with our findings, Dimeski and Jones [9] reported no significant differences between ultracentrifugation and high-speed centrifugation, using metrological criteria based on the coefficient of variation of data for sodium ion, creatinine, urate, protein, lactate dehydrogenase, and magnesium.

We found differences in protein, calcium, and aspartate aminotransferase in the comparison of high-speed centrifugationa and 1,1,2-trichlorotrifluoroethane, and in protein, albumin, and calcium in the comparison of high-speed centrifugation and LipoClear. LipoClear and 1,1,2-trichlorotrifluoroethane were both not suitable for lipemia removal in serum/plasma samples when testing for protein and calcium. This may be because of incomplete removal of the interference by these reagents. The use of lipemia removal methods other than centrifugation-based methods may involve the introduction of a reagent that might interfere with the measurement of certain biochemical parameters. Our results do not support the use of LipoClear, in agreement with those of Saracevic et al [12], who concluded that LipoClear is not suitable for lipemia removal from samples to measure concentrations of glucose, sodium ion, potassium ion, chloride ion, phosphorus, magnesium, creatine kinase MB, alkaline phosphatase, gamma-glutamyl transferase, protein, albumin, and C-reactive protein. In addition, 1,1,2-trichlorotrifluoroethane has been found to interfere with the measurement of certain biochemical parameters, including gamma-glutamyl transferase, C-reactive protein, and creatine kinase MB [11, 12]. In our study, 1,1,2-trichlorotrifluoroethane did not interfere with glutamyl transferase measurements.

Thus, high-speed centrifugation at 10,000 $\times g$ for 15 minutes is appropriate for lipemia removal in serum/plasma samples. LipoClear and 1,1,2-trichlorotrifluoroethane are not suitable because they interfere with the measurement of certain biochemical parameters.

\section{Authors' Disclosures of Potential Conflicts of Interest}

The authors declare that they have no conflicts of interest.

\section{Acknowledgment}

We would like to thank Cristina Ruiz Iruela for participating in the processing of removing lipemia in her training period in Bellvitge University Hospital and especially acknowledge the Sociedad Española de Química Clínica (SEQC).

\section{REFERENCES}

1. Kroll M. Evaluating interference caused by lipaemia. Clin Chem 2004; 
50:1968-9.

2. Farrell $C L$ and Carter $A C$. Serum indices : managing assay interference. Ann Clin Biochem 2016;53:527-38.

3. Ryder KW, Glick MR, (Ascp) M, Glick SJ. Incidence and amount of turbidity, hemolysis, and icterus in serum from outpatients. Lab Med 1991;22:415-8.

4. Nikolac N. Lipaemia: causes, interference mechanisms, detection and management. Biochem Med 2014;24:57-67.

5. Goldenberg NM, Wang P, Glueck CJ. An observational study of severe hypertriglyceridemia, hypertriglyceridemic acute pancreatitis, and failure of triglyceride-lowering therapy when estrogens are given to women with and without familial hypertriglyceridemia. Clin Chim Acta 2003; 332:11-9.

6. Calmarza P and Cordero J. Lipaemia interferences in routine clinical biochemical tests. Biochem Med 2011;21:160-6.

7. Walker PL and Crook MA. Lipaemia: causes, consequences and solutions. Clin Chim Acta 2013;418:30-2.

8. Dimeski G. Interference testing. Clin Biochem Rev 2008;29(S1):S43-8.

9. Dimeski $\mathrm{G}$ and Jones BW. Lipaemic samples: effective process for lipid reduction using high speed centrifugation compared with ultracentrifugation. Biochem Med (Zagreb) 2011;21:86-92.

10. CLSI. Hemolysis, icterus, and lipaemia/turbidity indices as indicators of interference in clinical laboratory analysis. 1st ed. CLSI C56. Wayne, PA: Clinical and Laboratory Standards Institute. 2011.

11. Vermeer HJ, Steen G, Naus JM, Goevaerts B, Pauline T, Schoenmakers $\mathrm{CHH}$. Correction of patient results for Beckman Coulter LX-20 assays affected by interference due to hemoglobin, bilirubin or lipids : a practical approach. Clin Chem Lab Med 2007;45:114-9.

12. Saracevic A, Nikolac N, Simundic A. The evaluation and comparison of consecutive high speed centrifugation and LipoClear ${ }^{\circledR}$ reagent for lipaemia removal. Clin Biochem 2014;47:309-14.

13. Bergmeyer HU, Hørder M, Rej R. Approved recommendation (1985) on IFCC methods for the measurement of catalytic concentration of enzymes. Part 3. IFCC method for alanine aminotransferase. J Clin Chem Clin Biochem 1986;24:481-95.

14. Bergmeyer HU, Hørder M, Rej R. Approved recommendation (1985) on IFCC methods for the measurement of catalytic concentration of enzymes. Part 2. IFCC Method for aspartate aminotransferase. J Clin Chem Clin Biochem 1986;24:497-510.

15. Tietz NW, Rinker AD, Shaw LM. International Federation of Clinical Chemistry. IFCC methods for the measurement of catalytic concentration of enzymes, Part 5. IFCC method for alkaline phosphatase (orthophosphoric-monoester phosphohydrolase, alkaline optimum, EC 3.1.3.1). J Clin Chem Clin Biochem 1983;21:731-48.

16. Szasz G. New substrates for measuring gamma-glutamyl-transpeptidase activity. Z Klin Chem Klin Biochem 1974;12:228.

17. Spanish Society of Clinical Biochemistry and Molecular Pathology. Criteria for the assessment of the analytical and clinical significance of interferences in clinical biochemistry. Quim Clínica 1995;14:107-9.

18. CLSI. Statistical quality control for quantitative measurement procedures: principles and definitions; approved guideline. 3rd Ed. CLSI C24A3. Wayne, PA: Clinical Laboratory Standards Institute. 2006. 